\title{
CLASSIFICATION OF SOME SIMPLE GRADED PRE-LIE ALGEBRAS OF GROWTH ONE
}

\author{
FRÉDÉRIC CHAPOTON
}

\begin{abstract}
We classify a class of infinite-dimensional simple graded pre-Lie algebras on the graded vector space underlying the algebra of Laurent polynomials, with a specific form for the product.
\end{abstract}

\section{Introduction}

The notion of pre-Lie algebra can be seen as a weakened form of associative algebra, still giving a Lie algebra by anti-symmetrization. It has been introduced by Gerstenhaber in his work on deformations of algebras [Ger64]. More recently, it has been studied from the point of view of operad theory and seen to be related to rooted trees [CL01].

The motivating problem for this paper is the classification of infinitedimensional simple graded pre-Lie algebras of finite growth over $\mathbb{C}$. This seems to be a difficult problem, as it implies in particular the same classification for associative algebras. The analogous problem for Lie algebras has been solved by Mathieu in [Mat91, Mat92]. The first step in the case of Lie algebras was the classification for growth not greater than 1 done in [Mat86b, Mat86a]. As a modest first step to the similar classification problem for pre-Lie algebras, we obtain here the classification of simple graded pre-Lie algebras such that

1) The underlying graded vector space is $E=\oplus_{i \in \mathbb{Z}} \mathbb{C} e_{i}$.

2) The product is given by $e_{i} \circ e_{j}=f(i) g(j) e_{i+j}$,

for some complex-valued functions $f$ and $g$ on $\mathbb{Z}$.

This Ansatz for the product has no special meaning, except that it allows for a full classification, which is unknown in the general case.

Theorem 0.1. Any simple graded pre-Lie algebra structure on $E$ of the form (\#) is isomorphic either to the algebra $A_{a}$ defined by

$$
e_{i} \circ e_{j}=(1+a i) e_{i+j}
$$

for some complex number $a$, or to the algebra $B_{b}$ defined by

$$
e_{i} \circ e_{j}=\frac{i}{1+b j} e_{i+j}
$$


for some complex number $b$ which is not the inverse of an integer.

All these algebras are pairwise non-isomorphic, with the exceptions $A_{a} \simeq$ $A_{-a}$ and $B_{b} \simeq B_{-b}$.

One can see that the associated Lie algebras are isomorphic to the Witt algebra $\mathrm{W}$, except for $A_{0}$ with trivial Lie algebra. The pre-Lie algebras $A_{a}$ and $B_{b}$ have been considered before by Kuperschmidt in his study of a pre-Lie ("quasiassociative") structure on the Virasoro algebra [Kup99]. There remains the possibility of other isomorphism classes of simple pre-Lie algebra structures on $E$ with no presentation fitting our Ansatz.

The first section collects basic general results on pre-Lie algebras. The second section contains the proof that any simple graded pre-Lie algebra fitting the Ansatz is isomorphic either to an algebra $A_{a}$ or an algebra $B_{b}$. In the third section, possible isomorphisms between the algebras $A_{a}$ and $B_{b}$ are determined. The last section deals with injective morphisms into pre-Lie algebras of vector fields on the line.

\section{General properties of simple pre-Lie algebras}

This section contains basic material on pre-Lie algebras, some of which can already be found in [Bur96, Bur98], which are concerned with finite dimensional pre-Lie algebras (under the name left-symmetric algebras).

Definition 1.1. A pre-Lie algebra is a vector space $L$ endowed with $\circ$ : $L \otimes L \rightarrow L$ satisfying

$$
(x \circ y) \circ z-x \circ(y \circ z)=(x \circ z) \circ y-x \circ(z \circ y) \quad \forall x, y, z \in L .
$$

An ideal in a pre-Lie algebra is a two-sided ideal for $\circ$.

Definition 1.2. A graded pre-Lie algebra with non-zero product and without any non-trivial homogeneous ideal is called simple.

Lemma 1.3. In a pre-Lie algebra $L$, the subspaces

$$
\begin{aligned}
\operatorname{Ann}(L) & =\{x \in L \mid \forall y \in L \quad y \circ x=0\} \\
\text { and } L \circ L & =\text { span of }\{x \circ y \mid x, y \in L\}
\end{aligned}
$$

are ideals and are preserved by any automorphism of $L$.

Proof. These subspaces are clearly invariant by any automorphism. The only non-trivial check is that $x \circ y$ is in $\operatorname{Ann}(L)$ for $x \in \operatorname{Ann}(L)$ and $y \in L$. Indeed, one has, for $z \in L$,

$$
z \circ(x \circ y)=z \circ(y \circ x)-(z \circ y) \circ x+(z \circ x) \circ y,
$$

which vanishes by definition of $\operatorname{Ann}(L)$.

In a graded pre-Lie algebra $L, \operatorname{Ann}(L)$ and $L \circ L$ are therefore homogeneous ideals. 
Lemma 1.4. In a simple graded pre-Lie algebra $L, \operatorname{Ann}(L)$ is zero and $L \circ L$ is $L$.

Proof. By simplicity, $\operatorname{Ann}(L)$ and $L \circ L$ can only be zero or $L$. If either $\operatorname{Ann}(L)$ is $L$ or $L \circ L$ is zero, then the product is zero, which is excluded by definition.

Lemma 1.5. Let $L$ be a graded pre-Lie algebra. If the associated graded Lie algebra is graded simple then $L$ is graded simple as a pre-Lie algebra.

Proof. A non-trivial homogeneous pre-Lie ideal would give a non-trivial homogeneous Lie ideal.

Lemma 1.6. Let e be an invariant vector in a pre-Lie algebra L, i.e. eox= 0 for all $x \in L$. Then the map $R_{e}: y \mapsto y \circ e$ is a derivation of the pre-Lie product of $L$.

Proof. Write the pre-Lie axiom for $(x \circ y) \circ e$.

\section{Reduction to cases $A$ and $B$}

The condition that formula (\#) defines a pre-Lie algebra can be stated as the vanishing of the numbers $\left(C_{i, j, k}\right)_{i, j, k \in \mathbb{Z}}$, where $C_{i, j, k}$ is the coefficient of $e_{i+j+k}$ in

$$
\left(e_{i} \circ e_{j}\right) \circ e_{k}-e_{i} \circ\left(e_{j} \circ e_{k}\right)-\left(e_{i} \circ e_{k}\right) \circ e_{j}+e_{i} \circ\left(e_{k} \circ e_{j}\right) .
$$

Remark that the numbers $C_{i, j, j}$ are always zero because $C_{i, j, k}=-C_{i, k, j}$. Explicitly, $C_{i, j, k}$ is given by

(8) $f(i)((f(i+j)-f(i+k)) g(j) g(k)+(f(k) g(j)-f(j) g(k)) g(j+k))$.

One can now use the general properties proved in the previous section to deduce properties of $f$ and $g$ from the simplicity of $E$.

$\operatorname{As} \operatorname{Ann}(E)$ is zero, the function $g$ can not vanish anywhere. One can (and will) in particular assume without restriction that $g(0)=1$ by dividing the basis and the function $g$ by $g(0)$.

As $E \circ E$ is $E$, the function $f$ cannot be identically zero. Let us discuss according to the value of $f(0)$.

2.1. Case A: $f(0) \neq 0$. When $f(0) \neq 0$, one can assume without restriction that $f(0)=1$ by dividing the basis and the function $f$ by $f(0)$.

Lemma 2.1. In this case, one has $g(k)=1$ for all $k$.

Proof. Write the condition $C_{0,0, k}=0$ and use that $g(k) \neq 0$.

Therefore the vanishing of formula (8) reduces to

$$
f(i)(f(i+j)-f(j)-(f(i+k)-f(k)))=0 .
$$


Let us introduce a family of simple graded pre-Lie algebras $A_{a}$ indexed by a complex number $a$. They are defined on the vector space $E$ by the formula $e_{i} \circ e_{j}=(1+a i) e_{i+j}$. One can check that, for all $a \neq 0$, the associated Lie algebra of $A_{a}$ is the Witt algebra $\mathrm{W}$, which is a simple graded Lie algebra. It follows by Lemma 1.5 that $A_{a}$ is a simple graded pre-Lie algebra for $a \neq 0$. The graded-simplicity of $A_{0}$ is easy to prove and the associated Lie algebra has zero product.

Proposition 2.2. Any simple graded pre-Lie algebra structure on E with product

$$
e_{i} \circ e_{j}=f(i) e_{i+j}
$$

for some complex-valued function $f$ on $\mathbb{Z}$ with $f(0)=1$, is isomorphic to one of the $A_{a}$.

Proof. Let us first assume that $f(i)=0$ for all $i \neq 0$. Then the subspace $\oplus_{i \neq 0} \mathbb{C} e_{i}$ is a non-trivial homogeneous ideal of $E_{f}$, which is a contradiction.

One can therefore assume that there exists $i \neq 0$ with $f(i) \neq 0$. We do the proof when there exists such a positive $i$, the negative case being strictly analog. Let $d$ be the smallest positive $i$ such that $f(i) \neq 0$ and let $f(d)=1+a$ for some complex number $a \neq-1$.

If $d=1$, then formula (9) for $i=1, j=0$ and $k$ implies that $f(k)=1+k a$ for all $k$. This is the pre-Lie algebra $A_{a}$ introduced above.

If $d>1$, then $f(1)=0$ and formula (9) for $i=d, j=0$ and $k=1$ implies that $f(d+1)=a$. If $a=0$ then $f$ is periodic of period $d$ and therefore

$$
\left\{\begin{array}{l}
f(i)=1 \text { if } d \mid i, \\
f(i)=0 \text { otherwise. }
\end{array}\right.
$$

In this case, the subspace $\bigoplus_{i \neq 0[d]} \mathbb{C} e_{i}$ is a non-trivial homogeneous ideal of $E_{f}$, which is a contradiction.

One can therefore assume that $d>1$ and $a \neq 0$. If $d=2$, the sequence of values of $f$ on non-negative integers starts with

$$
1,0,1+a, a, 1+2 a, \ldots,
$$

and $a \neq 0$ implies $1+2 a=a-1$, i.e. $a=-2$ and $f(k)=1-k$ for all $k$. This is the pre-Lie algebra $A_{-1}$.

The only unsettled case is when $d \geq 3$ and $a \neq 0$. Then the sequence of values of $f$ on non-negative integers starts with

$$
1, \underbrace{0,0, \ldots}_{d-1 \text { zeroes }}, 1+a, a, a, \ldots,
$$

and $a \neq 0$ implies $a=a-1$, which is a contradiction. 
2.2. Case B: $f(0)=0$. When $f(0)=0$, there exists $i \neq 0$ such that $f(i) \neq 0$. The vector $e_{0}$ is invariant, so it gives a derivation by Lemma 1.6. The vanishing of $C_{i, j, 0}$ is then equivalent to the following equation:

$$
f(i)(f(i+j)-f(i)-f(j))=0 .
$$

Let us introduce a family of simple graded pre-Lie algebras $B_{b}$ for a complex number $b$ which is not the inverse of an integer. They are defined on the vector space $E$ by the formula $e_{i} \circ e_{j}=i /(1+b j) e_{i+j}$. By the change of basis $\bar{e}_{i}=(1+b i) e_{i}$, this is equivalent to

$$
\bar{e}_{i} \circ \bar{e}_{j}=\frac{i(1+b i)}{1+b(i+j)} \bar{e}_{i+j},
$$

which is the formula given by Kuperschmidt [Kup99].

One can check, by using formula (15), that the associated Lie algebra of $B_{b}$ is the Witt algebra $\mathrm{W}$ for all $b$. It follows by Lemma 1.5 that $B_{b}$ is a simple graded pre-Lie algebra for any admissible $b$.

Proposition 2.3. Any simple graded pre-Lie algebra structure on $E$ with product

$$
e_{i} \circ e_{j}=f(i) g(j) e_{i+j},
$$

for some complex-valued functions $f, g$ on $\mathbb{Z}$ with $f(0)=0$, is isomorphic to one of the $B_{b}$.

Proof. There exists $i \neq 0$ such that $f(i) \neq 0$. Assume for example that there exists a positive such $i$ and let $d$ be the smallest positive integer such that $f(d) \neq 0$. Then one can assume without restriction that $f(d)=d$, by dividing the basis and $f$ by $f(d) / d$. It follows from (14) that $f(i)-i$ is a periodic function of $i$ with period $d$. It has also period $d+1$, because $f(d+1)$ is either $d$ or $2 d$, which is non-zero. This implies that $f(i)-i$ is constant. As $f(0)=0$, one has $f(i)=i$ for all $i$.

Let us define a complex-valued function on $\mathbb{Z}$ by $h(i)=1 / g(i)$. The vanishing of (8) becomes

$$
k(h(j+k)-h(k))=j(h(j+k)-h(j)) .
$$

As a special case, $h(j)+h(-j)=2$ for all $j$. Let $h(1)=1+b$ for some complex number $b$. Then $h(-1)=1-b$. One proves by recursion that $h(j)=1+b j$ for all $j$.

To summarize, we have obtained the following law:

$$
e_{i} \circ e_{j}=\frac{i}{1+b j} e_{i+j},
$$

which is defined only if $b$ is not the inverse of an integer, and is the pre-Lie algebra $B_{b}$ introduced above. 


\section{Isomorphism types}

In this section, the algebras $A_{a}$ and $B_{b}$ are not considered as graded, i.e. one works in the category of not necessarily graded pre-Lie algebras.

The map $e_{i} \mapsto e_{-i}$ defines an isomorphism between $A_{a}$ and $A_{-a}$. The map $e_{i} \mapsto-e_{-i}$ defines an isomorphism between $B_{b}$ and $B_{-b}$.

A useful remark is that $A_{0}$ is the only associative algebra among the $A_{a}$ and $B_{b}$. In fact, $A_{0}$ is isomorphic to the commutative algebra $\mathbb{C}\left[x, x^{-1}\right]$ of Laurent polynomials.

Proposition 3.1. The only non-trivial (not necessarily graded) isomorphisms between the $A_{a}$ and $B_{b}$ pre-Lie algebras are the above isomorphisms $A_{a} \simeq A_{-a}$ and $B_{b} \simeq B_{-b}$.

Before proving the proposition, we state a crucial lemma. Let $L$ be a pre-Lie algebra which is one of the $A_{a}$ or $B_{b}$.

Lemma 3.2. Let $v \in L$. If $R_{v}: x \mapsto x \circ v$ is a locally finite endomorphism of $L$, then $v$ is proportional to $e_{0}$.

Proof. One can assume that $v \neq 0$. Let $v=\sum_{i=k}^{\ell} \lambda_{i} e_{i}$ with $\lambda_{k}$ and $\lambda_{\ell}$ non-zero. Let us show that $\ell>0$ implies that $R_{v}$ is not locally finite.

Let $N$ be a large positive integer. Consider the sequence

$$
e_{N}, R_{v}\left(e_{N}\right), R_{v}^{2}\left(e_{N}\right), \ldots
$$

By recursion, one can see that for all $p \geq 0, R_{v}^{p}\left(e_{N}\right)$ is a finite sum of the form $\sum_{j \leq N+p \ell} \mu_{j} e_{j}$ with $\mu_{N+p \ell} \neq 0$, provided that $N$ is larger than $-1 / a$ in the case of the $A_{a}$ algebra. Therefore these vectors are linearly independent. This is a contradiction with local finiteness of $R_{v}$. Hence we can assume that $\ell \leq 0$. By the same proof with reverse grading, one can assume that $k \geq 0$, so that $v \in \mathbb{C} e_{0}$.

Let us go back to the proof of proposition 3.1.

Proof. Let $L$ be a pre-Lie algebra which is one of the $A_{a}$ or $B_{b}$. By Lemma 3.2 , the subspace $\mathbb{C} e_{0}$ is invariant by any automorphism. Let $v$ be a non-zero multiple of $e_{0}$. We now discuss according to the vanishing of $v \circ v$.

If $v \circ v=0$, then the algebra must be one of the $B_{b}$. The eigenspace decomposition of $R_{v}$ gives back the homogeneous decomposition, up to reversal of the grading. Choose one of the two opposite gradings and let $w \neq 0$ be in $\mathbb{C}_{1}$. Then $(w \circ w) \circ w$ and $w \circ(w \circ w)$ are two non-zero vectors in $\mathbb{C} e_{3}$. Their proportionality coefficient gives back $b$ or $-b$, according to the choice of grading.

If $v \circ v \neq 0$, then the algebra must be one of the $A_{a}$. One can further assume that $a \neq 0$, because $A_{0}$ is the only associative algebra among the $A_{a}$. Then $e_{0}$ is the unique vector in $\mathbb{C} e_{0}$ such that $e_{0} \circ e_{0}=e_{0}$. The eigenvalues 
of $R_{e_{0}}$ give back the sequence $1+a i$ up to a choice of grading. So we recover $a$ up to sign.

\section{Vector fields realizations}

We determine here all realizations of the algebras $A_{a}$ and $B_{b}$ as subalgebras of the pre-Lie algebra $V_{1}(\Omega)$ of analytic complex vector fields on an connected open subset $\Omega$ of the complex affine line $\mathrm{A}^{1}(\mathbb{C})$.

Let us start with the $A$ case:

Proposition 4.1. The algebra $A_{a}$ for $a \neq 0$ is isomorphic to the subalgebra of $V_{1}\left(\mathbb{C} \backslash \mathbb{R}^{-}\right)$with basis

$$
e_{i}=x^{1+a i} \frac{\partial}{\partial x}
$$

Up to an affine change of variable, this is the unique realization of $A_{a}$ as a pre-Lie algebra of vector fields on the line.

There is no injection of $A_{0}$ into the pre-Lie algebra of analytic vector fields on an open subset of $\mathrm{A}^{1}(\mathbb{C})$.

Proof. Let $\psi$ be a map from $A_{a}$ to such a pre-Lie algebra. Because of $e_{0} \circ e_{0}=e_{0}, \psi\left(e_{0}\right)$ must belong to $\mathbb{C} \partial_{x} \oplus \mathbb{C} x \partial_{x}$ with non-vanishing component in $\mathbb{C} x \partial_{x}$. One can assume by a translation of the variable $x$ that $\psi\left(e_{0}\right)$ belongs to $\mathbb{C} x \partial_{x}$. Then, in fact, $\psi\left(e_{0}\right)=x \partial_{x}$. Because $e_{i} \circ e_{0}=(1+a i) e_{i}$, $\psi\left(e_{i}\right)$ is in $\mathbb{C} x^{1+a i} \partial_{x}$ for all $i$. Hence one must restrict to a simply-connected open subset of $\mathbb{C}^{*}$, and injectivity is only possible if $a \neq 0$. By a dilatation of the variable $x$, one can assume that $\psi\left(e_{1}\right)=x^{1+a} \partial_{x}$. Then is is easy to see that necessarily $\psi\left(e_{i}\right)=x^{1+a i} \partial_{x}$ for all $i$. Finally, it is easy to check that $\psi$ is a pre-Lie algebra map.

Now, consider the $B$ case:

Proposition 4.2. The algebra $B_{0}$ is isomorphic to the subalgebra of $V_{1}(\mathbb{C})$ with basis

$$
e_{i}=\exp (i x) \frac{\partial}{\partial x}
$$

Up to an affine change of variable, this is the unique realization of $B_{0}$ as a pre-Lie algebra of vector fields on the line.

There is no injection of $B_{b}$ into the pre-Lie algebra of analytic vector fields on an open subset of $\mathrm{A}^{1}(\mathbb{C})$ for $b \neq 0$.

Proof. Let $\psi$ be a map from $B_{b}$ to such a pre-Lie algebra. Because of $e_{0} \circ e_{0}=$ $0, \psi\left(e_{0}\right)$ must be in $\mathbb{C} \partial_{x}$. By a dilatation of the variable $x$, one can assume that $\psi\left(e_{0}\right)=\partial_{x}$. Then $e_{i} \circ e_{0}=i e_{i}$ implies that $\psi\left(e_{i}\right)$ is in $\mathbb{C} \exp (i x) \partial_{x}$ for all $i$. By a translation of the variable $x$, one can assume that $\psi\left(e_{1}\right)=\exp (x) \partial_{x}$. Then one can check that $\psi\left(e_{1}\right) \circ \psi\left(e_{-1}\right)=-\psi\left(e_{-1}\right) \circ \psi\left(e_{1}\right)$, which implies 
$b=0$. It is easy then to see that $\psi\left(e_{i}\right)=\exp (i x) \partial_{x}$ for all $i$. This indeed defines a pre-Lie algebra map.

\section{References}

[Bur96] Dietrich Burde. Left-invariant affine structures on reductive Lie groups. J. Algebra, 181(3):884-902, 1996.

[Bur98] Dietrich Burde. Simple left-symmetric algebras with solvable Lie algebra. Manuscripta Math., 95(3):397-411, 1998.

[CL01] Frédéric Chapoton and Muriel Livernet. Pre-Lie algebras and the rooted trees operad. Internat. Math. Res. Notices, (8):395-408, 2001.

[Ger64] Murray Gerstenhaber. On the deformation of rings and algebras. Ann. of Math. (2), 79:59-103, 1964.

[Kup99] Boris A. Kupershmidt. On the nature of the Virasoro algebra. J. Nonlinear Math. Phys., 6(2):222-245, 1999.

[Mat86a] Olivier Mathieu. Classification des algèbres de Lie graduées simples de croissance $\leq 1$. Invent. Math., 86(2):371-426, 1986.

[Mat86b] Olivier Mathieu. Sur un problème de V. G. Kac: la classification de certaines algèbres de Lie graduées simples. J. Algebra, 102(2):505-536, 1986.

[Mat91] Olivier Mathieu. Classification of simple graded Lie algebras of finite growth. In Proceedings of the International Congress of Mathematicians, Vol. I, II (Kyoto, 1990), pages 799-806, Tokyo, 1991. Math. Soc. Japan.

[Mat92] Olivier Mathieu. Classification of simple graded Lie algebras of finite growth. Invent. Math., 108(3):455-519, 1992.

Received Received date / Revised version date

\section{LACIM}

UnIVERsité DU QuÉBEC À MonTrÉAL

CP 8888 SUCC. CENTRE VILLE

MONTRÉAL QUÉBEC H3C 3P8

CANADA

E-mail address: chapoton@math.uqam.ca 